\title{
Essai d'analyse des relations génétiques entre les races bovines françaises à l'aide du polymorphisme biochimique*
}

\author{
F Grosclaude ${ }^{1}$, RY Aupetit ${ }^{2}$, J Lefebvre ${ }^{3}$, JC Mériaux ${ }^{2}$ \\ 1 INRA, laboratoire de génétique biochimique \\ 2 INRA, laboratoire des groupes sanguins \\ 3 INRA, laboratoire de génétique factorielle, centre de recherches de Jouy-en-Josas, \\ 78350 Jouy-en-Josas, France
}

(Reçu le 18 octobre 1989; accepté le $1^{\text {er }}$ juin 1990)

Résumé - Des méthodes d'analyse phylogénétique et multivariate (indices de distance génétique $D$ et $D_{m}$ de Nei, $f_{\theta}$ de Cavalli-Sforza, $d_{o}$ de Gregorius, méthode UPGMA de Sneath et Sokal, analyse des composantes principales et des données centrées) ont été mises en œuvre pour tenter de préciser les relations génétiques existant entre 18 races bovines françaises, auxquelles avait été adjointe la race britannique Shorthorn en raison des introductions de son ancêtre Durham en France au siècle dernier. Les calculs ont porté sur les fréquences géniques à 13 locus polymorphes, dont 11 locus de groupes sanguins érythrocytaires, le locus de la transferrine sérique et celui de la caséine $\beta$.

Cette étude conduit à distinguer 4 sous-ensembles de races: 1) le groupe des races du Nord: Frisonne Pie-Noire, Flamande, Maine-Anjou, Shorthorn; 2) le groupe des races du Centre et du Sud-Ouest : Charolaise, Ferrandaise, Limousine, Salers, Aubrac, Blonde d'Aquitaine; 3) un groupe comprenant à la fois des races de l'Ouest : Bretonne Pie-Noire, Parthenaise, et de l'Est : Vosgienne, Montbéliarde, Pie-Rouge de l'Est, Brune, Abondance et Tarine; 4) la seule race Normande. Ces subdivisions se raccordent de manière cohérente aux rameaux correspondants de l'arbre phylogénétique des races européennes de Manwell et Baker (1980).

Les aspects les plus inattendus de ces résultats sont l'apparentement de la race Charolaise avec les races blondes du Sud-Ouest, plutôt qu'avec les races jurassiennes, ainsi que le regroupement des races Bretonne Pie-Noire et Parthenaise avec les races de l'Est, qui rappelle toutefois des éléments de la classification de Dechambre (1913).

Les plus fortes variances entre races des fréquences géniques s'observent aux locus de groupes sanguins érythrocytaires $C, S$, puis $A$, ainsi qu'au locus de la caséine $\beta$. Dans l'analyse en composantes principales, ce sont également des allèles des locus $C, A$ et $\beta-C n$ qui donnent les valeurs les plus élevées du coefficient de corrélation $(0,85$ à 0,98$)$ avec les 3 premières composantes. L'analyse des données centrées fait apparaître un parallélisme étroit entre les évolutions, en fonction de la première variable canonique, des fréquences

* Ce travail est dédié à la mémoire de Pierre Charlet (1912-1982), Professeur de Zootechnie à l'Institut National Agronomique de Paris. 
de $C^{C_{1}}$ et de $\beta-C n^{A^{1}}$, qui se font sensiblement selon un gradient sud-nord, ainsi qu'entre celles, en sens inverse, de $C^{C_{1}^{\prime \prime}}$ et de $\beta-C n^{A^{2}}$.

bovin / races françaises / race Shorthorn / polymorphisme biochimique / distance génétique / phylogénie

Summary - Tentative analysis of genetic relationship between French cattle breeds using biochemical polymorphism. Methods of phylogenetic and multivariate analysis (measures of genetic distance $\mathrm{D}$ and $\mathrm{D}_{\mathrm{m}}$ of Nei, $\mathrm{f}_{\theta}$ of Cavalli-Sforza, $\mathrm{d}_{\mathrm{o}}$ of Gregorius, UPGMA method of Sneath and Sokal, principal components analysis) were carried out for a tentative clarification of the relationship between 18 French breeds. Those methods were applied using gene frequencies calculated for 13 polymorphic loci, including 11 blood group loci, the locus of blood serum transferrin, and that of $\beta$-casein. The British Shorthorn was also included in the analysis because its Durham ancestor had been introduced to some French regions during the last century.

The results permit a subdivision to be made of the 19 breeds into 4 subsets: 1) a group of Northern breeds: Frisonne Pie-Noire, Flamande, Maine-Anjou, Shorthorn; 2) Central and South-Western breeds: Charolaise, Ferrandaise, Limousine, Salers, Aubrac, Blonde d'Aquitaine; 3) Western and Eastern breeds: Bretonne Pie-Noire, Parthenaise, and Vosgienne, Montbéliarde, Pie-Rouge de l'Est, Brune, Abondance, Tarine; 4) the Normande breed alone. Those subdivisions may be linked in a coherent manner with branches of the phylogenetic tree for European cattle breeds, which was proposed by Manwell and Baker (1980).

Unexpectedly, the Charolaise is connected with the South-Western Blonde breeds rather than with the Simmental type cattle. The association of the Bretonne Pie-Noire and Parthenaise with the Eastern breeds is in accordance with the classification suggested by Dechambre (1913).

The highest between-breed variances of gene frequencies were observed for the $\mathrm{C}, \mathrm{S}$ and A blood group loci, as well as at the $\beta$-casein locus. In the principal components analysis, alleles of the $\mathrm{C}, \mathrm{A}$ and $\beta$-casein loci also gave the highest values for the correlation coefficient (0.85-0.98) with the 3 first components. Centred data analysis brought out a close parallelism between the evolution, as a function of the first canonical variable, of the frequencies for $\mathrm{C}^{\mathrm{C}^{1}}$ and $\beta-\mathrm{Cn}^{\mathrm{A}^{1}}$, increasing along a south to north axis, and of those for $\mathrm{C}^{\mathrm{C}_{1}^{\prime \prime}}$ and $\beta-\mathrm{Cn}^{\mathrm{A}^{2}}$, decreasing along the same axis.

cattle / French breeds / British Shorthorn breed / biochemical polymorphism / genetic distance / phylogeny

\section{INTRODUCTION}

La création des livres généalogiques des races bovines françaises, qui marque la naissance officielle de ces races, s'est faite à partir de 1864 (Charolaise) et, pour la majorité d'entre elles, entre 1880 et 1914. La diversité du peuplement bovin de notre pays dans cette seconde moitié du XIX ${ }^{e}$ siècle est illustrée par la monographie de Sanson (1884) qui ne répertorie pas moins de 36 «variétés», rattachées à 6 grands groupes d'extension européenne. En 1963, l'ouvrage de Quittet distingue encore 30 races d'effectifs il est vrai très inégaux (de 3 millions d'animaux pour la race Normande à quelques dizaines de milliers pour les races Bleue du Nord, Froment du Léon, Villard de Lans, Pyrénées centrales et Mézenc). On a assisté depuis à une évolution spectaculaire, dominée par l'ascension de la race Frisonne Pie-Noire, elle-même progressivement envahie par les gènes de la souche Holstein (Colleau et $a l, 1982)$, et par le déclin accéléré d'une série d'autres races. Parmi les plus petites, certaines ont déjà disparu (Bordelaise, Mézenc); d'autres ne comptaient plus, en 
1987, que quelques centaines, voire quelques dizaines d'animaux: Bretonne PieNoire: 456; Ferrandaise: 217; Villard-de-Lans:125; Froment du Léon: 27; etc (Avon, 1989).

Pour tenter d'enrayer cette tendance, des programmes de conservation ont été mis en place avec, depuis 1976, une aide financière du ministère de l'Agriculture; ils concernent actuellement une quinzaine de races de petits ou très petits effectifs (Avon, 1989). Le fait que chacune de ces interventions représente un certain coût amène naturellement à s'interroger sur le choix des races qualifiées pour en bénéficier. Il paraît judicieux de concentrer les efforts sur celles qui présentent, par rapport aux races dominantes, un réel degré d'originalité. Les questions qu'on se pose dans ce domaine suscitent un nouvel intérêt pour les recherches concernant l'origine des races et leurs relations de parenté.

Il existe toute une série de monographies consacrées aux races bovines françaises, dues pour les unes à des chercheurs de profession (Vissac, 1970, pour l'Aubrac, Bougler et Le Liboux, 1973, pour la Charolaise, etc), pour les autres à des amateurs (Vernier, 1953, pour la Montbéliarde, Sarrazin, 1962 pour la Normande, etc). Toutefois, pour ce qui concerne le classement des races françaises selon leurs origines, 2 auteurs seulement, Sanson (1884) et Baron, cité par Dechambre (1913), ont, selon Denis (1983), «fait œuvre originale et scientifique». Le classement de Sanson se fonde sur des mesures craniométriques, celui de Baron sur un ensemble de caractères morphologiques (profil crânien, proportions, format, secondairement phanères et aptitudes).

La variabilité génétique existant dans les populations peut être mesurée par des techniques de plus en plus diverses et performantes. On dispose en particulier de données assez complètes sur le polymorphisme des groupes sanguins, de certaines protéines sériques et des protéines du lait dans nombre de races bovines. Ces données ont été mises à profit pour tenter de préciser les relations génétiques existant entre races autrichiennes (Kidd et Pirchner, 1971), ibériques (Kidd et al, 1982; Gonzalez et al, 1987), italiennes (Astolfi et al, 1983), allemandes (Graml et al, 1986).

Pour ce qui est des études dépassant le cadre d'un seul pays, Kidd (1969) a tenté de préciser les relations existant entre 18 races d'origines diverses, n'incluant comme race française que la seule Charolaise. Plus récemment, en se basant sur les fréquences alléliques à 10 locus polymorphes de protéines du sang et du lait, Manwell et Baker (1980) ont établi l'arbre phylogénétique de 10 groupes raciaux (3 zébus, 7 taurins) définis (Baker et Manwell, 1980) à partir de critères indépendants de l'information biochimique. Les 7 groupes taurins considérés par ces auteurs sont définis comme suit: 1) Nord-Européen: races scandinaves et anglaises; 2) Pie des Plaines: Pie-Rouge et Pie-Noire de la zone nord du continent européen; 3) Brachyceros rouge européenne incluant en principe la Flamande; 4) Brachyceros des îles de la Manche: incluant la Normande et les races bretonnes; 5) Brachyceros des hauteurs (Pie-rouge de l'Est, Montbéliarde, Brune des Alpes, Tarentaise, nombreuses races de l'Europe centrale et du pourtour méditerranéen; 6) mixte Primigenius-Brachyceros: Charolaise, Limousine, Blonde d'Aquitaine, Parthenaise, autres races du Sud de l'Europe; 7) Primigenius : races à longues cornes ibériques et podoliques.

Or, Manwell et Baker montrent que, sous certaines hypothèses assez vraisemblables, cet arbre phylogénétique se superpose à la carte géographique de telle manière 
que les 10 groupes raciaux de taurins ou zébus se placent dans des zones remarquablement proches de leur centre présumé d'origine et de diversification. C'est ainsi qu'en plaçant le foyer du groupe nord-européen en Écosse, celui du groupe Pie des Plaines près de la Baltique, à la frontière germano-polonaise, et celui du groupe des îles de la Manche sur ces îles, la branche principale de l'arbre phylogénétique suit le bassin du Danube, le centre du groupe Primigenius se place près de la Podolie, celui du groupe mixte Primigenius-Brachyceros vers la côte yougoslave, celui du groupe Brachyceros des hauteurs sur l'Autriche occidentale et celui du groupe Brachyceros rouge en Pologne méridionale (Manwell et Baker, 1980). Ainsi, même si certains aspects peuvent être discutés (mode de découpage des groupes raciaux, point de divergence entre rameaux Primigenius et Brachyceros localisé sur la côte bulgare et non en Elam et Sumer comme le propose Epstein, 1971), le travail de ces auteurs aboutit à des résultats d'une cohérence assez frappante.

Aucune analyse d'ensemble n'a été effectuée jusqu'ici sur les races françaises, qui ont seulement fait l'objet de 2 études partielles, celle de Grosclaude et Millot (1962) sur la race Montbéliarde, et celle de Bouquet et Grosclaude (1968) sur la race Flamande, qui comporte également des données sur la Frisonne Pie-Noire. Le présent travail tente d'établir, à partir des données relatives à 13 systèmes polymorphes, les relations génétiques existant entre 18 races actuellement représentées en France, auxquelles a été adjointe la Shorthorn anglaise (anciennement Durham) compte tenu de son introduction, au siècle dernier, dans certaines de nos races.

\section{MATÉRIEL ET MÉTHODES}

\section{Systèmes génétiques polymorphes}

L'analyse fait appel au polymorphisme génétique des 11 systèmes de groupes sanguins érythrocytaires connus chez les bovins $\left(A, B, C, F, J, L, M, S, Z, R^{\prime}, T^{\prime}\right)$, de la transferrine sérique $(T f)$ et de la caséine $\beta(\beta-C n)$ (tableau I). Ces 13 systèmes sont génétiquement indépendants (Larsen, 1971). Pour éliminer l'incidence de la recombinaison au sein des 2 systèmes complexes $B$ et $C$, qui sont vraisemblablement codés l'un et l'autre par plusieurs gènes étroitement liés (Grosclaude et al, 1979; Guérin et al, 1981), le polymorphisme de ces systèmes a été simplifié et ramené à ce qui peut être assimilé à de véritables séries alléliques. Le système $C$ est ainsi réduit à un ensemble tétra-allélique, par regroupement des phénogroupes (haplotypes) comportant respectivement les facteurs antigéniques $C_{1}, C_{2}, C_{1}^{\prime \prime}$ et $C_{2}^{\prime \prime}$, dont les déterminants génétiques sont très proches, voire allèles, selon la carte du système établie par Guérin et al (1981). De même, le système $B$ a été réduit à 6 allèles par regroupement des phénogroupes comportant respectivement $G_{1}, I_{1}, K, P_{1}, T, J^{\prime \prime}$, dont les déterminants génétiques sont également très proches selon la carte proposée par Grosclaude et al(1983), un septième allèle représentant les phénogroupes ne comportant aucun de ces facteurs. Toutefois, dans certaines races, 2 de ces facteurs antigéniques peuvent coexister dans quelques phénogroupes rares $(f<0,005)$; les phénogroupes comportant $I_{1} K$ et $G_{1} K$ ont été arbitrairement classés avec le groupe allélique $(K), I_{1} T$ avec $(T)$ et $I_{1} G_{1}$ avec $\left(G_{1}\right)$. L'ensemble allélique ainsi défini avec les 13 systèmes génétiques retenus compte au total 51 allèles, soit 38 variables statistiquement indépenciantes. 
Tableau I. Systèmes génétiques et allèles pris en compte.

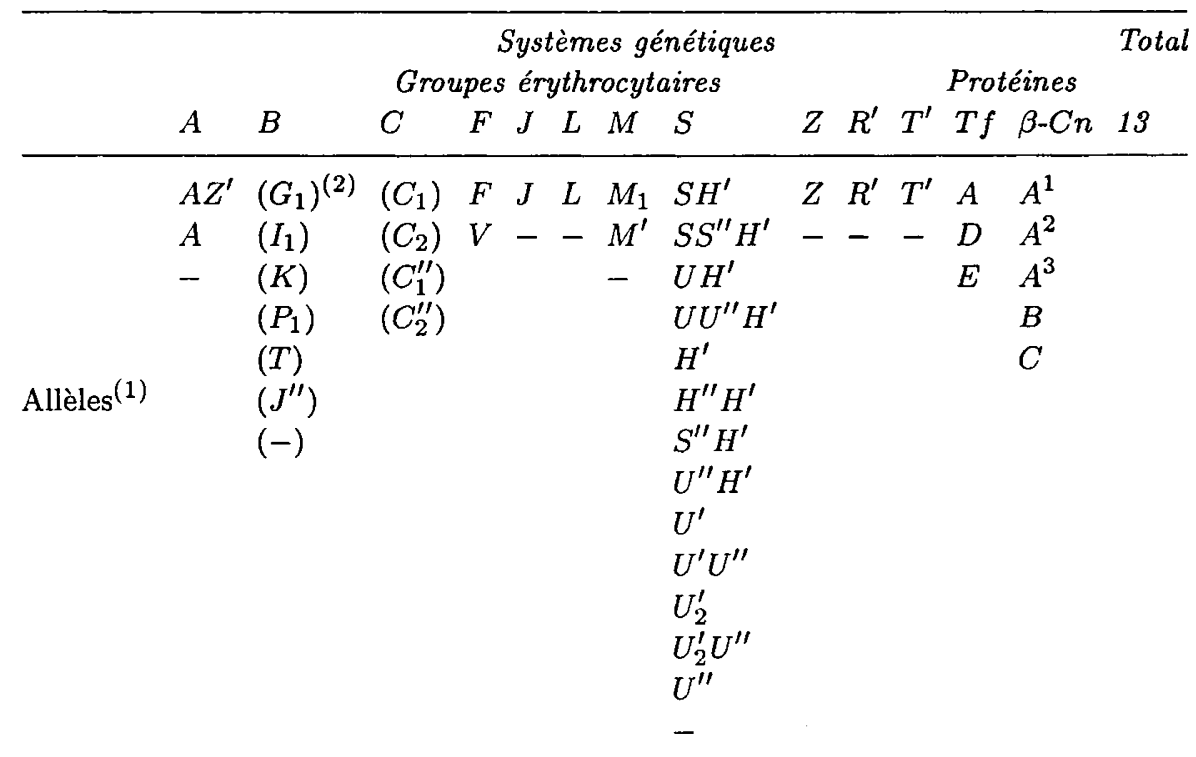

Nombre

\begin{tabular}{lllllllllllllll} 
d'allèles & 3 & 7 & 4 & 2 & 2 & 2 & 3 & 14 & 2 & 2 & 2 & 3 & 5 & 51 \\
\hline
\end{tabular}

(1) Lire: $A^{A Z^{\prime}}, A^{A}, A^{-}, B^{\left(G_{1}\right)}$, etc; -: désigne un allèle négatif. (2) $\left(G_{1}\right),\left(I_{1}\right) \ldots$ $\left(C_{2}^{\prime \prime}\right)$ : ensemble des allèles déterminant les phénogroupes (haplotypes) possédant le facteur antigénique $G_{1}, I_{1} \ldots C_{2}^{\prime \prime}$

\section{Techniques d'analyse}

La description des réactifs de groupes sanguins utilisés et celle de la technique d'analyse sont données en détail dans Grosclaude et al (1979). A noter que le facteur antigénique $F 16$ a pris, depuis, l'appellation de $J^{\prime \prime}$. Le polymorphisme de la transferrine a été mis en évidence par électrophorèse en gel d'amidon selon Ashton (1965), celui de la caséine $\beta$ par électrophorèse en gel d'amidon et d'acrylamide, comme décrit par Grosclaude et al (1974).

\section{Races}

Le tableau II donne la liste des 19 races considérées, dont l'implantation géographique, selon Quittet (1963), est schématisée dans la figure 6. Certaines d'entre elles sont d'origine étrangère relativement récente, et toujours alimentées d'apports génétiques étrangers (Frisonne Pie-Noire, Brune, Pie-Rouge de l'Est). La race Shorthorn anglaise, autrefois Durham, a été incluse dans l'étude compte tenu de son utilisation plus ou moins importante, au siècle dernier, dans certaines de nos races (de 
Tableau II. Constitution des échantillons raciaux.

\begin{tabular}{|c|c|c|c|c|}
\hline \multirow[t]{2}{*}{ Races } & \multirow[t]{2}{*}{ Abréviations } & \multicolumn{3}{|c|}{ Effectifs et dates des échantillons } \\
\hline & & $\begin{array}{l}\text { Groupes } \\
\text { érythrocytaires }\end{array}$ & Transferrine & Caséine $\beta$ \\
\hline Abondance & $A B$ & $211(1966-70)$ & $133(1965)$ & $127(1985)$ \\
\hline Aubrac & $A U$ & $156(1966-70)$ & $52(1965)$ & $94(1982)$ \\
\hline Blonde d'Aquitaine & $B L$ & $284(1980-83)$ & $123(1965-68)$ & $161(1968)$ \\
\hline Bretonne Pie-Noire & $B R$ & $91(1966-81)$ & $66(1966)$ & $83(1985)$ \\
\hline Brune & $B U$ & $350(1967-73)$ & $39(1965)$ & $157(1979)$ \\
\hline Charolaise & $\mathrm{CH}$ & $435(1983)$ & $118(1965)$ & $51(1973)$ \\
\hline Ferrandaise & $F E$ & $39(1979-82)$ & $29(1982)$ & $81(1979)$ \\
\hline Flamande & $F L$ & $400(1963)$ & $400(1963)$ & $298(1971)$ \\
\hline Frisonne Pie-Noire & $F R$ & $450(1963)$ & $450(1965)$ & $280(1965)$ \\
\hline Limousine & $L I$ & $377(1984)$ & $108(1965)$ & $40(1973)$ \\
\hline Maine-Anjou & $M A$ & $196(1978-83)$ & $79(1965)$ & 39 (1973) \\
\hline Montbéliarde & $M O$ & $396(1973)$ & $379(1965)$ & $350(1965)$ \\
\hline Normande & NO & $454(1977-79)$ & $133(1965)$ & $318(1972)$ \\
\hline Parthenaise & $P A$ & $139(1966-81)$ & $124(1966-77)$ & $96(1966)$ \\
\hline Pie-Rouge de l'Est & $P R$ & $185(1972-75)$ & $123(1965)$ & $142(1979)$ \\
\hline Salers & $S A$ & $226(1977-82)$ & $54(1986)$ & $103(1979)$ \\
\hline Tarentaise & $T A$ & 271 (1967-73) & $51(1965)$ & $287(1967)$ \\
\hline Vosgienne & $\mathrm{VO}$ & $317(1975)$ & $493(1975)$ & $228(1975)$ \\
\hline Shorthorn & $S H$ & $63(1981)$ & $61(1967)^{(1)}$ & 381 (1981) \\
\hline
\end{tabular}

(1) Selon Rausch et al, 1967.

Lapparent, 1914). Cinq autres races pour lesquelles les données restent incomplètes n'ont pas été prises en considération (Bazadaise, Corse, Froment du Léon, Gasconne, Villard de Lans).

\section{Echantillonnage}

La collecte de données en vue de ce travail s'est étalée de 1963 à 1986 (tableau II). Les échantillons d'animaux ont été uniquement constitués de femelles adultes, les résultats concernant les pères et les produits ne servant qu'à l'interprétation des génotypes maternels. Dans une même race, les prélèvements de sang et de lait ont souvent été obtenus à partir d'échantillons différents. Dans le cas des systèmes sanguins, les données proviennent soit de sondages spécifiques (races Frisonne PieNoire et Vosgienne par exemple), soit des fichiers du service d'analyse des groupes sanguins (contrôles de filiations). Pour les races de grand effectif (Normande, Charolaise, Montbéliarde, Limousine, etc) l'échantillon est alors un extrait de l'ensemble des données existantes, constitué de manière aussi représentative que possible pour l'aire d'extension principale de la race et pour une période bien définie; pour les races de faible effectif, il peut par contre représenter l'ensemble des résultats disponibles, souvent cumulés sur plusieurs années, et correspond parfois à une bonne partie des animaux encore inscrits au Livre généalogique de la race (Ferrandaise, Bretonne Pie-Noire, Vosgienne). Seuls ont été publiés jusqu'ici des résultats sur les races Flamande et Frisonne Pie-Noire (Bouquet et Grosclaude, 1968), ainsi que 
sur la caséine $\beta$ (Grosclaude, 1988). Les prélèvements de sang et de lait de la race Shorthorn proviennent d'élevages dispersés en Angleterre (du Yorkshire au nord au Somerset au sud) et au pays de Galles (Dyfed).

\section{Détermination des fréquences alléliques}

Les fréquences alléliques ont été calculées, comme dans Bouquet et Grosclaude (1968), par comptage direct pour les systèmes génétiques où tous les allèles sont codominants $(F, T f, \beta-C n)$, par la méthode de la racine carrée pour les systèmes bi- ou tri-alléliques comportant un allèle «négatif » $\left(A, J, L, M, Z, R^{\prime}, T^{\prime}\right)$ et par la méthode itérative de Ceppellini et al (1956) pour les systèmes complexes $(B, C$ et $S$ ).

\section{Distances génétiques}

Quatre des différentes formules proposées dans la littérature pour mesurer la distance génétique entre 2 populations à partir de fréquences géniques ont été appliquées, parallèlement, aux données de cette étude: l'indice de distance standard, $D$, de Nei (1972), l'indice de distance minimale, $D_{m}$, du même auteur (Nei, 1973) et l'indice $F_{\theta}$ de Cavalli-Sforza (1969), lesquels ont été couramment utilisés par d'autres auteurs, ainsi que l'indice de distance «absolue», $d_{o}$, de Gregorius (1984). Si $r$ est le nombre de locus considérés, $m_{j}$ le nombre d'allèles au $j^{e}$ locus, $x_{i j}$ et $y_{i j}$ les fréquences du $i^{e}$ allèle au $j^{e}$ locus dans les populations $X$ et $Y$, ces 4 indices de distance s'écrivent comme suit :

$$
D=-\log _{e}\left(J_{x y} / \sqrt{J_{x} J_{y}}\right)
$$

avec $J_{X}=\frac{1}{r} \sum_{j=1}^{r} \sum_{i=1}^{m_{j}} x_{i j}^{2}, J_{Y}=\frac{1}{r} \sum_{j=1}^{r} \sum_{i=1}^{m_{j}} y_{i j}^{2} ; J_{X Y}=\frac{1}{r} \sum_{j=1}^{r} \sum_{i=1}^{m_{j}} x_{i j} y_{i j}$

$$
\begin{aligned}
& D_{m}=\frac{1}{2 r} \sum_{j=1}^{r} \sum_{i=1}^{m_{j}}\left(x_{i j}-y_{i j}\right)^{2} \\
& f_{\theta}=\frac{4 \sum_{j=1}^{r}\left(1-\sum_{i=1}^{m_{j}} \sqrt{x_{i j} y_{i j}}\right)}{\sum_{j=1}^{r}\left(m_{j}-1\right)} \\
& d_{o}=\frac{1}{2 r} \sum_{j=1}^{r} \sum_{i=1}^{m_{j}}\left|x_{i j}-y_{i j}\right|
\end{aligned}
$$

Selon Gregorius (1984), un indice de distance génétique doit remplir plusieurs conditions: 
- avoir des propriétés métriques, en particulier satisfaire au «principe d'inégalité du triangle» $(D(X, Y) \leqslant D(X, Z)+D(Z, Y))$;

- donner une valeur maximale, de 1 , lorsque $\Sigma x_{i} y_{i}=0$;

- ne dépendre que des allèles communs aux 2 populations comparées;

- donner une valeur décroissant linéairement jusqu'à 0 lorsqu'on déplace linéairement une population vers une autre.

L'indice $d_{o}$ satisfait à toutes ces conditions, contrairement aux autres indices qui, en particulier, ne sont pas métriques. Toutefois Nei et al (1983) soulignent que la non-métricité de $D$ est due au caractère stochastique des substitutions géniques, et que $D$ est asymptotiquement métrique. Par ailleurs, si $D_{m}$ et $d_{o}$ tendent bien vers $1, f_{\theta}$ tend vers 4 et $D$ n'est pas borné. Il est bien connu que ces différentes formules, appliquées aux mêmes données, peuvent amener à des conclusions différentes. A titre d'exemple, $f_{\theta}$ est fortement sensible au nombre d'allèles de faible fréquence; pour les mêmes populations, sa valeur tend donc à varier avec la taille des échantillons (Nei et al, 1983). De manière générale, on peut penser que les discordances entre indices dans les résultats s'observeront plutôt pour les races au statut le plus incertain, comme celles situées à l'interface de 2 groupes phylogénétiques différents.

La représentation en dendrogrammes des relations entre races a été établie par la méthode «UPGMA» de Sneath et Sokal (1973).

\section{Calculs}

Les calculs ont été effectués sur ordinateur IBM 3083 EX à l'aide de programmes existants (M Nei) ou créés à cet effet. L'analyse des coordonnées principales (Gower, 1966) de la matrice des distances obtenues avec la formule de Gregorius et celle des données centrées (Lefebvre, 1983) ont été effectuées sur micro-ordinateur WANG 2200 LVP à l'aide des programmes de Lefebvre et al (1981). L'analyse en composantes principales a été effectuée sur ordinateur IBM 3083 EX à l'aide du logiciel SAS (SAS Institute Inc, Cary NC, 27511, USA).

\section{RÉSULTATS}

\section{Hétérogénéité des fréquences alléliques entre races}

La figure 1 donne, pour chacun des $m \rightarrow 1$ allèles indépendants de chaque locus, la valeur du rapport de Wahlund, $\frac{\sigma^{2}}{\bar{f}(1-\bar{f})}$ où $\bar{f}$ est la moyenne et $\sigma^{2}$ la variance des fréquences alléliques dans les races considérées. Ce rapport mesure le degré d'hétérogénéité entre ces races. Les plus fortes valeurs de ce paramètre s'observent pour des allèles des locus des groupes érythrocytaires $C$ et $S$, et du locus de la caséine $\beta$, et, en second lieu, pour des allèles des locus de groupes érythrocytaires $A$ et $B$. Certains de ces allèles $\left(C^{C_{1}}, C^{C_{1}^{\prime \prime}}, S^{U^{\prime}}, \beta-C n^{A^{1}}, \beta-C n^{A^{2}}, A^{A}\right)$ se rencontrent dans toutes les races et peuvent atteindre des fréquences élevées. 


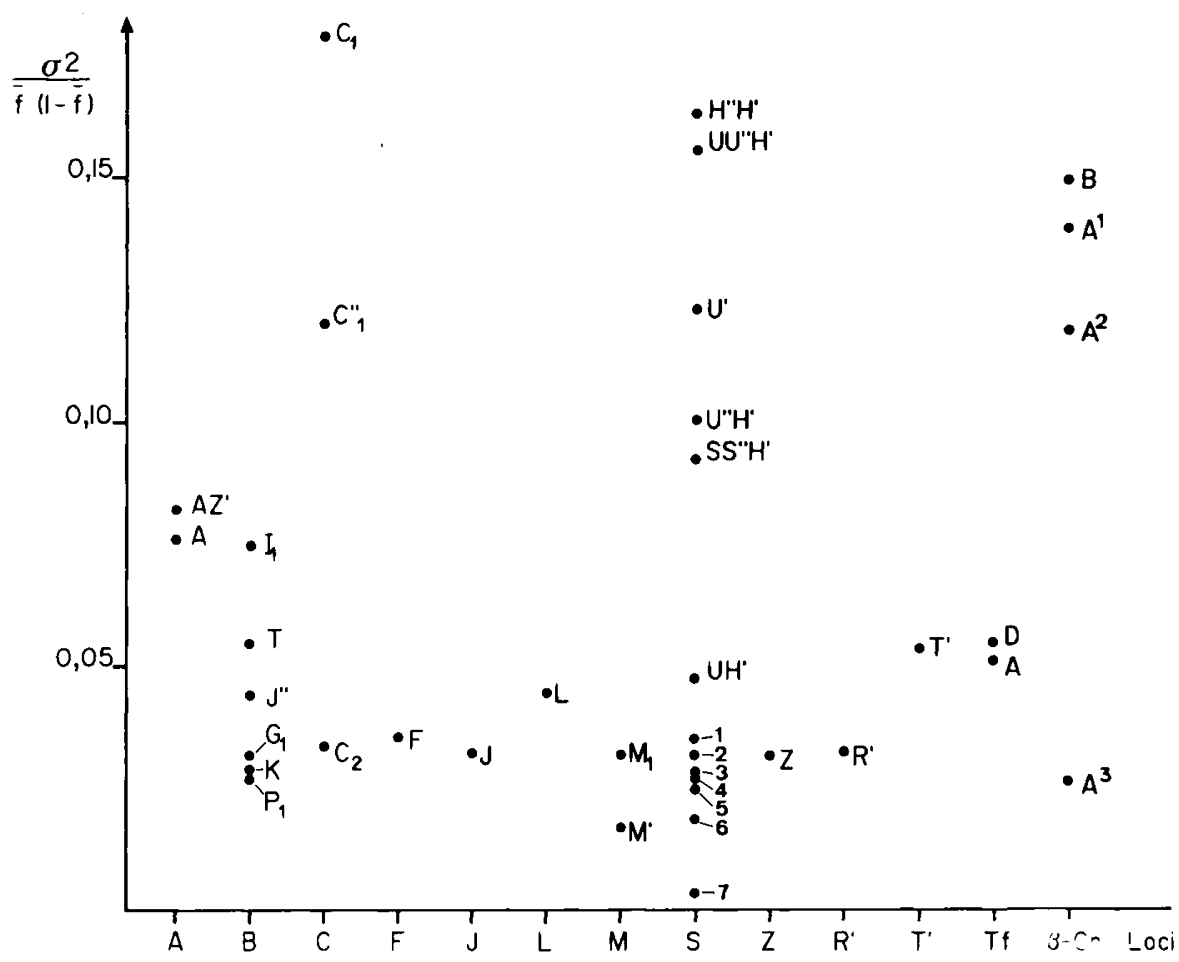

Fig 1. Valeurs du rapport de Wahlund, $\sigma^{2} / \bar{f}(1-\bar{f})$, pour les $m-1$ allèles indépendants de chacun des 13 locus pris en compte. Au système $S, 1=S^{\prime \prime} H^{\prime} ; 2=H^{\prime} ; 3=S H^{\prime}$; $4=U_{2}^{\prime} U^{\prime \prime} ; 5=U^{\prime \prime} ; 6=U_{2}^{\prime} ; 7=U^{\prime} U^{\prime \prime}$.

\section{Relations génétiques entre races}

La figure 2 donne les représentations en dendrogrammes obtenues à partir des matrices des distances génétiques calculées à l'aide des indices $D, D_{m}, d_{o}$ et $f_{\theta}$. On constate que $D_{m}$ et $d_{o}$ conduisent à des résultats très voisins qui amènent à distinguer 4 groupes de races:

- un groupe de races du Nord (Maine-Anjou, Flamande, Frisonne Pie-Noire, Shorthorn);

- un groupe de races du Centre et du Sud-Ouest (Charolaise, Ferrandaise, Blonde d'Aquitaine, Aubrac, Salers, Limousine);

- un groupe comprenant des races de l'Est (Vosgienne, Montbéliarde, Brune, PieRouge de l'Est, Tarine, Abondance) et de l'Ouest (Bretonne Pie-Noire, Parthenaise) que nous appellerons groupe de l'axe est-ouest;

- la race Normande seule.

Les résultats les plus inattendus, sont l'apparentement de la race Charolaise avec les races blondes (Blonde d'Aquitaine, Limousine) et non avec les races jurassiennes comme le voudrait la littérature, ainsi que la proximité génétique, au sein du groupe 

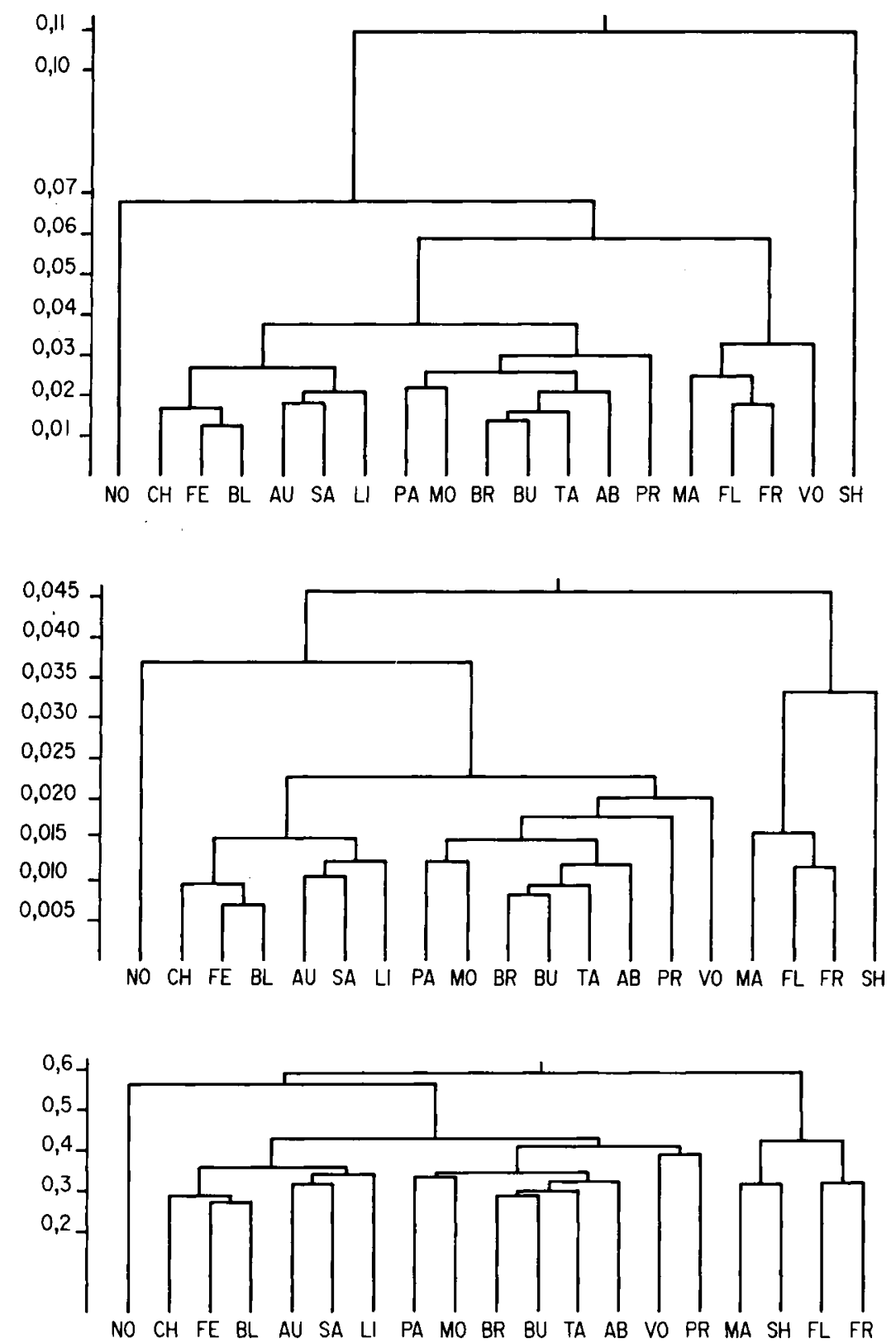

Fig 2a, b et c. 


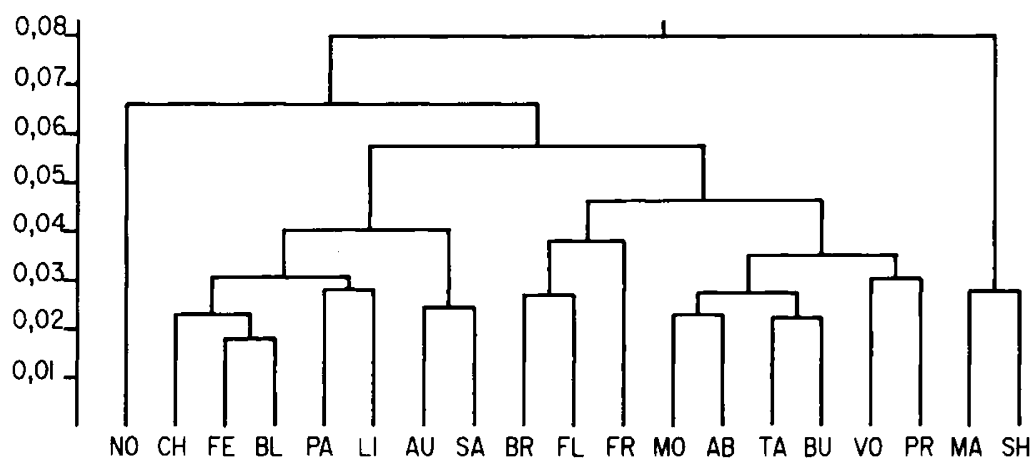

Fig. 2d.

Fig 2. Dendrogrammes obtenus à partir des valeurs données par les indices de distance $D(a)$ et $D_{m}(b)$ de Nei, $d_{o}$ de Gregorius $(c)$, et $f_{\theta}$ de Cavalli-Sforza $(d)$. Rappel des désignations des races: $N O$, Normande; $C H$, Charolaise; $F E$, Ferrandaise; $B L$, Blonde d'Aquitaine; $A U$, Aubrac; $S A$, Salers; $L I$, Limousine; $P A$, Parthenaise; $M O$, Montbéliarde; $B R$, Bretonne Pie-Noire; $B U$, Brune; $T A$, Tarine; $A B$, Abondance; $P R$, Pie-Rouge de l'Est; $M A$, Maine-Anjou; $F L$, Flamande; $F R$, Frisonne Pie-Noire; $V O$, Vosgienne; $S H$, Shorthorn.

de l'axe est-ouest, de la Bretonne Pie-Noire avec la Brune et la Tarine. L'indice $D$ donne un dendrogramme peu différent où la Shorthorn s'éloigne toutefois des autres races et où la Vosgienne se rattache au groupe des races du Nord. Enfin, à côté d'une similitude d'ensemble, l'indice $f_{\theta}$ conduit à quelques différences qui concernent notamment la position des 2 races de l'Ouest, Parthenaise et Bretonne Pie-Noire:

- la Parthenaise se retrouve ici dans le groupe des races du Centre et du SudOuest, proche de la Limousine;

- la Bretonne Pie-Noire est proche de la Flamande et de la Frisonne Pie-Noire, ces 3 races se rattachant ensuite au groupe des races de l'Est.

Par ailleurs, par rapport à $D_{m}$ et $d_{o}, f_{\theta}$ conduit à détacher la Flamande et la Frisonne Pie-Noire de la Maine-Anjou et la Shorthorn, ces 2 dernières restant bien séparées de l'ensemble des autres races.

La figure 3a donne la représentation des résultats de l'analyse en coordonnées principales de la matrice des distances obtenues avec l'indice $d_{o}$, qui est, rappelonsle, une mesure métrique. La figure $3 \mathrm{~b}$ donne les résultats de l'analyse en composantes principales à partir de la matrice des variances et covariances des fréquences alléliques. Les conclusions de cette analyse sont très voisines de celles obtenues avec les indices de distance $d_{o}$ et $D$. On note, en particulier, que la Parthenaise est proche de la Montbéliarde, et que la Bretonne Pie-Noire est presque accolée à la Brune.

\section{Analyse du pouvoir discriminant des allèles}

Dans l'analyse en composantes principales, les 3 premières composantes rendent compte de $72 \%$ de la variabilité totale (tableau III). On note les très fortes corrélations existant entre ces composantes et des allèles des systèmes $C\left(1^{\text {re }}\right.$ composante), $A$ ( $2^{\mathrm{e}}$ composante) et $\beta-C n$ ( $1^{\mathrm{re}}$ et $3^{\mathrm{e}}$ composantes). A l'opposé, 

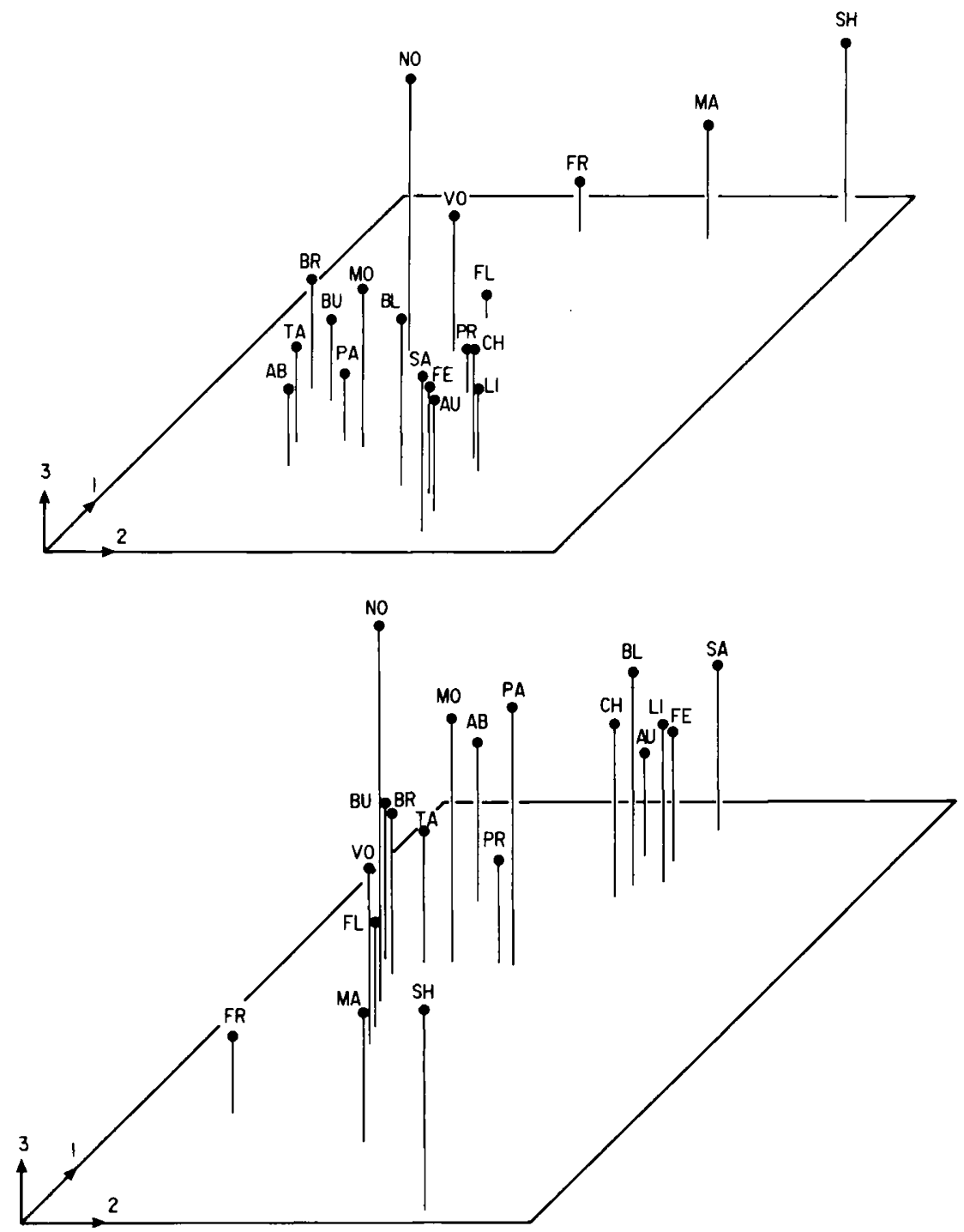

Fig 3. a) Analyse en coordonnées principales de la matrice des valeurs données par l'indice de distance $d_{o}$ de Gregorius. b) Analyse en composantes principales de la matrice des variances et covariances des fréquences géniques. Cette représentation, très voisine de celle de la figure 3a, est donnée sous un autre angle (image de miroir). Désignation des races comme dans la figure 2 .

le système $B$ fait partie des systèmes dont les allèles conduisent, pour le coefficient de corrélation avec les composantes principales, à des valeurs relativement faibles. L'analyse des données centrées, en permettant une représentation simultanée des observations et des variables, fait ressortir les liaisons, même non linéaires, entre 


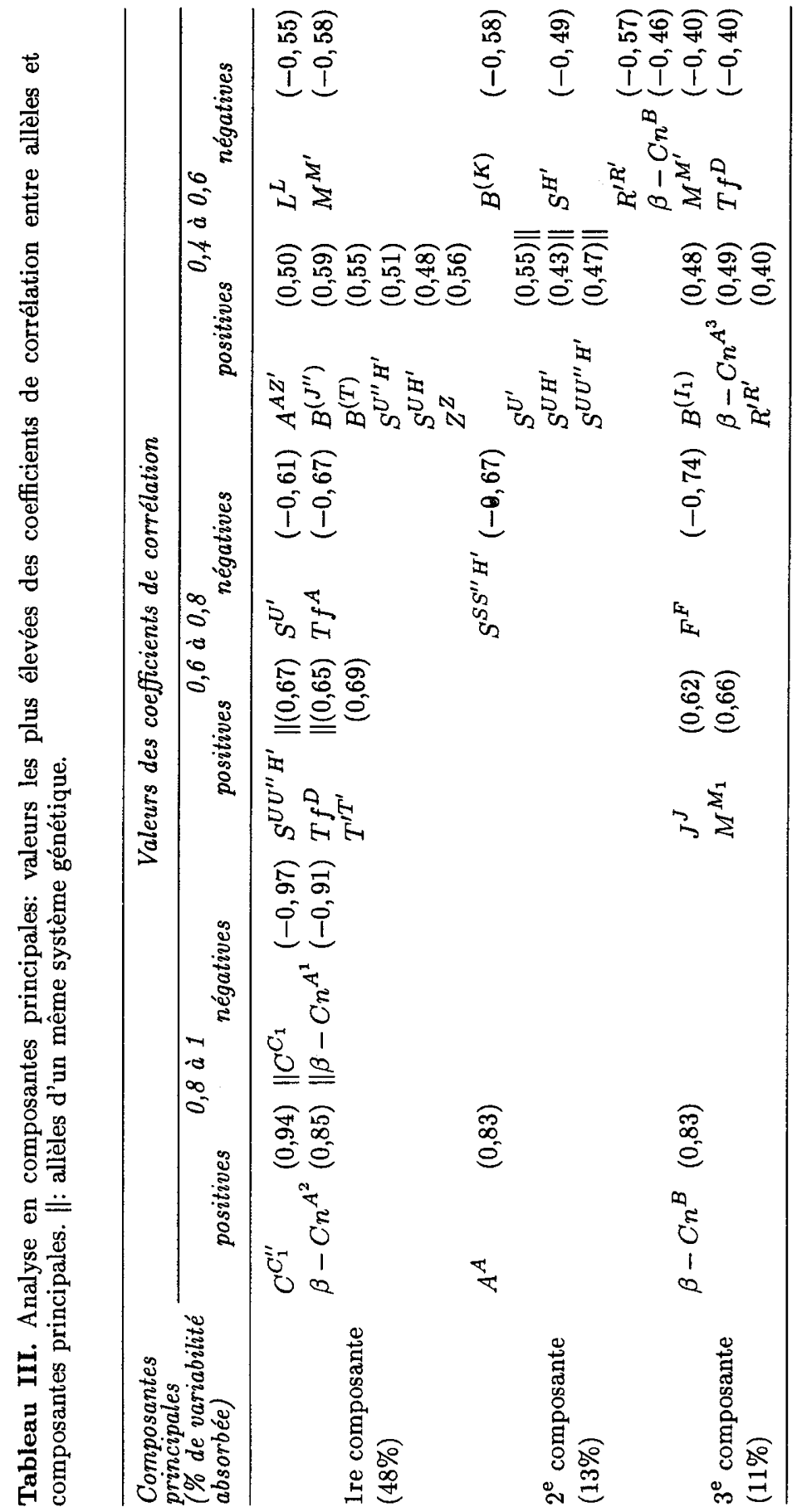




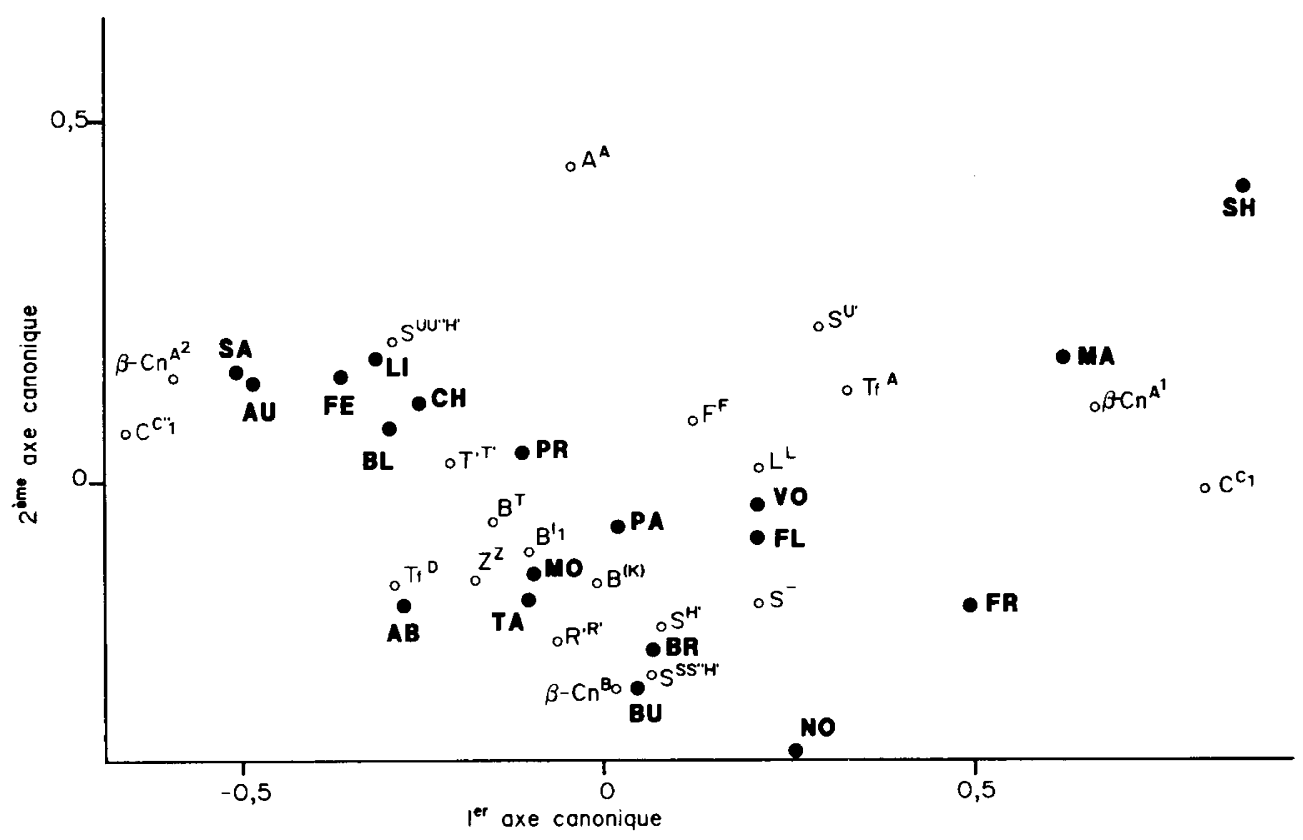

Fig 4. Analyse des données centrées: projection, sur le plan des 2 premiers axes canoniques, des races désignées comme dans la figure 2 , et des allèles les plus discriminants.

celles-ci, ainsi que l'influence des divers allèles sur les positions des races (fig 4). Les 3 premiers axes canoniques rendent compte respectivement de $47,8 \%, 12,9 \%$ et $10,4 \%$ de la variabilité totale ( $71,1 \%$ pour l'ensemble). Aux 2 extrémités du premier axe, se projettent d'une part $C^{C_{1}^{\prime \prime}}$ et $\beta-C n^{A^{2}}$, d'autre part $C^{C_{1}}$ et $\beta-C n^{A^{1}}$. Sur cet axe, Salers et Aubrac sont très proches, et près du premier groupe de variables, Shorthorn et Maine-Anjou près du second groupe. La figure 5 traduit le remarquable parallélisme entre l'évolution, en fonction de la première variable canonique, de $C^{C_{1}}$ et $\beta-C n^{A^{1}}$, ainsi que celle, en sens inverse, et encore plus étroite, de $C^{C_{1}^{\prime \prime}}$ et $\beta-C n^{A^{2}}$. Le deuxième axe est déterminé essentiellement (fig 4) par $A^{A}$, opposé à $\beta$ $C n^{B}, S^{S S^{\prime \prime} H^{\prime}}, R^{R^{\prime}}$ et $S^{H^{\prime}}$. Sur le troisième axe (non représenté) $\beta-C n^{B}$, puis $A^{A}$, $T f^{A}$ et $J^{J}$ s'opposent à $F^{F}, T F^{D}$ et $\beta-C n^{A^{2}}$. La Normande s'y détache nettement, en raison surtout de sa forte fréquence de $\beta-C n^{B}$. Par contre, l'étagement sur le troisième axe des races Frisonne Pie-Noire, Maine-Anjou et Shorthorn (voir fig 3a) est dû à leurs différences de fréquences pour $T f^{A}, A^{A}$ et $J^{J}$; ce sont essentiellement les mêmes allèles qui discriminent les races Aubrac et Salers sur ce troisième axe.

\section{DISCUSSION}

L'analyse des relations génétiques entre les races bovines françaises doit être située dans le cadre plus général de l'histoire des bovins en Europe, qui commence avec la révolution agricole du néolithique. En se basant sur la diffusion de céréales cultivées 


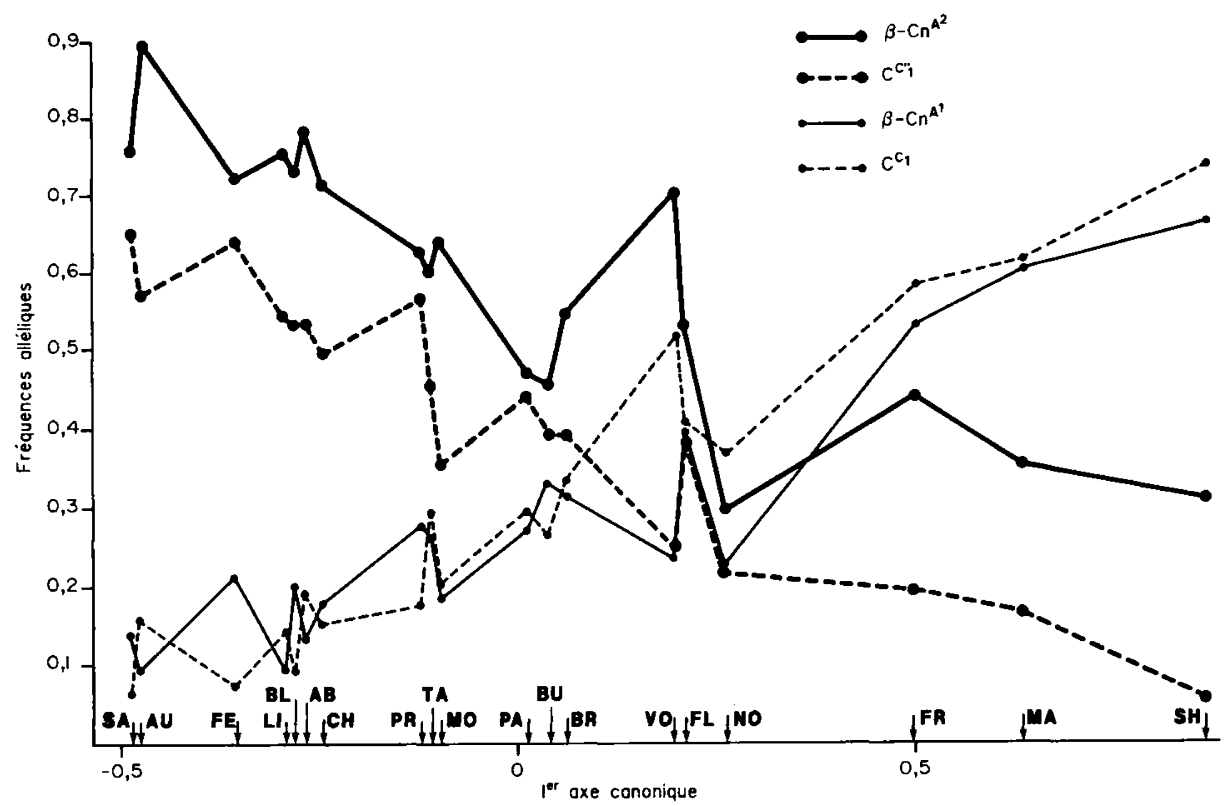

Fig 5. Analyse des données centrées: évolution des fréquences des allèles $C^{C_{1}}, C^{C_{1}^{\prime \prime}}, \beta$ $C n^{A^{1}}$ et $\beta-C n^{A^{2}}$ en fonction de la première variable canonique. La projection de chaque race sur le premier axe canonique est indiquée par une flèche.

(blé et orge), Ammerman et Cavalli-Sforza (1971) ont conclu que l'extension de l'agriculture s'était faite, à partir du Proche-Orient, de 8000 à 5500 environ avant J-C, selon un processus de vague d'avancée ("wave of advance") entretenu par la poussée démographique, au rythme moyen de $1 \mathrm{~km} / \mathrm{an}$. A côté d'une diffusion sur tout le pourtour méditerranéen, la progression humaine du néolithique en Europe s'est déployée suivant un axe orienté du sud-est vers le nord-ouest, en empruntant au départ la voie danubienne (Dolukhanov, 1971). On pense que la vague néolithique humaine a absorbé progressivement la population mésolithique préexistante. Dans l'hypothèse où les 2 populations présentaient, au départ, des différences de fréquences géniques, le processus d'absorption devait se traduire par la mise en place d'un gradient de fréquences caractérisé, au foyer d'origine, par des valeurs plus proches de celles du groupe néolithique primitif, et, à la périphérie, par des valeurs rappelant celles du groupe mésolithique. L'analyse en composantes principales de l'information collectée pour 38 allèles indépendants, appartenant à 10 locus (groupes sanguins, histocompatibilité, protéines sanguines), met bien en évidence un gradient principal, orienté du sud-est vers le nord-ouest (Menozzi et al, 1978). Comme les chances d'observer les effets génétiques d'une migration dépendent notamment du rapport du nombre des colonisateurs à celui des colonisés, on peut penser, selon Menozzi et al (1978) que, de toutes les migrations connues, l'expansion néolithique a été l'une de celles où ce rapport a été le plus élevé. 
Selon Epstein (1971) et Epstein et Mason (1984), les premiers bovins domestiqués l'ont été, vers 6000 avant J-C, soit dans les Balkans à partir de l'auroch européen (Bos primigenius primigenius), soit plus vraisemblablement en Asie du Sud-Ouest (Mésopotamie) à partir de l'auroch asiatique (Bos primigenius namadicus). Il s'agissait d'animaux à longues cornes, ressemblant beaucoup à des types encore actuels (Highland d'Ecosse, bétail à longues cornes du Portugal, bétail gris des steppes), et qui se sont répandus tout au long des rives de la Méditerranée en même temps que le type humain méditerranéen. Plus tard, vraisemblablement dans le courant du $4^{\mathrm{e}}$ millénaire avant J-C, des bovins à cornes courtes (Bos Brachyceros) ont été sélectionnés, sans doute à partir des précédents, dans la même région (Sumer, Elam), à l'occasion d'une révolution urbaine poussant au développement de la production laitière. Ces bovins à cornes courtes ont ensuite envahi l'Europe, et, dès 3000 avant $\mathrm{J}-\mathrm{C}$, ils ont prédominé sur les bovins à longues cornes, si l'on en juge par les vestiges archéologiques des palafittes suisses.

Fait remarquable, le travail de Manwell et Baker (1980), présenté dans l'introduction, dégage un axe de diffusion des bovins domestiques orienté du sud-est vers le nord-ouest qui se superpose à l'axe de diffusion de l'agriculture ainsi qu'au gradient de fréquences principal des populations humaines européennes. On aboutit ainsi à un ensemble de conclusions d'une cohérence assez frappante. Toutefois, compte tenu des dates proposées pour la diffusion de l'agriculture (8000 à 5500 avant J-C) et la domestication des bovins (6000 à 4000 avant J-C), l'avancée des bovins domestiques en Europe a été nettement postérieure à celle de la première agriculture (céréales cultivées). En fait, ces deux expansions, parties de la même région, ont dû, tout naturellement, emprunter la même voie de pénétration.

La littérature consacrée aux origines des races françaises est un mélange plus ou moins inextricable de mythes, d'hypothèses vraisemblables et de faits avérés. Au registre des mythes, on citera le rôle supposé de bétail amené par les légions romaines dans la création de la race bovine Charolaise (Friend, 1978), comme d'ailleurs dans celle d'une race équine - d'une autre région - la Boulonnaise (Quittet et Blanc, 1974). De même, selon Denis (1983), l'origine scandinave de la race Normande pourrait bien être un mythe ayant pris naissance au $19^{\mathrm{e}}$ siècle. En effet, comme l'apport viking n'aurait été que de $\ll 500$ colons pour une population préexistante de 100000 Celtes, Saxons, Francs et Méditerranéens», on peut s'interroger a fortiori sur l'influence génétique des bovins qu'ils ont pu amener. En fait, il est déjà difficile d'apprécier les conséquences génétiques de faits reconnus. On ne prendra pour exemple que l'incertitude qui règne sur l'impact réel des introductions de reproducteurs Durham effectuées dans certaines races il n'y a guère qu'un siècle à un siècle et demi. Enfin le souci de respecter des standards de race, à la même époque, s'est traduit par une sélection en faveur de certains caractères extérieurs (couleur, morphologie de la tête) qui a pu accentuer, entre races, des ressemblances ou des dissemblances, et conduire ainsi à une mauvaise appréciation du degré de parenté vrai entre leurs ensembles géniques respectifs.

Il est certain que la création des races actuelles n'a pas été un processus simple, et il est difficile de préciser quand, comment et avec quel degré le processus de divergence entre races a été brouillé par des phénomènes inverses de fusion ou d'immigration. La représentation des relations entre races par un dendrogramme est donc un choix simplificateur qui comporte le risque d'aboutir, sur certains 
points, à des conclusions inexactes. Par ailleurs, si les biais d'échantillonnage sont probablement réduits, compte tenu des précautions prises dans la constitution des échantillons, le nombre de locus pris en compte (13), quoique comparable à celui de beaucoup d'autres études, est relativement limité (Nei et al, 1983). Dans ces conditions, les résultats de notre analyse doivent être interprétés avec prudence. On considérera donc surtout les regroupements de races, plutôt que la structure détaillée des dendrogrammes. A noter que l'inclusion de la race Shorthorn constituait un test de fiabilité, compte tenu des certitudes que l'on a sur le rôle important qu'elle a joué, au siècle dernier, dans la création du bétail Maine-Anjou. Le regroupement observé de la Shorthorn et de la Maine-Anjou rassure donc sur la fiabilité de la méthode, tout au moins pour des événements relativement récents.

L'appartenance de la Charolaise au groupe du Centre et du Sud-Ouest peut surprendre, puisque cette race est généralement considérée comme d'origine jurassienne. Toutefois, selon Bougler et Le Liboux (1973), la Charolaise s'est progressivement différenciée à partir de la population à robe blanche dominante qui occupait le Nord de la France jusqu'aux limites du Jura. Les autres rameaux de cette population (Bressane, Fémeline) ont depuis été absorbés, principalement par la race Pie-Rouge de l'Est. Or la race Fémeline, dont l'aire d'extension se situait à l'est de celle de la Charolaise, donc plus près des races jurassiennes, est classée par Dechambre (1913) avec les races blondes (groupe des races convexilignes médiolignes). Le rattachement de la race Charolaise au groupe du Centre et du Sud-Ouest paraît solidement étayé par nos résultats. Parmi les éléments qui caractérisent ce groupe figure la fréquence élevée du couple d'allèles $S^{U H^{\prime}}$ et $S^{U U^{\prime \prime} H^{\prime}}$ déterminant le facteur $U$ (Charolaise $=0,34$; Blonde d'Aquitaine: 0,32; Aubrac: 0,31; Salers: 0,29; Limousine: 0,25; Ferrandaise: 0,24) contre 0,04 en Pie-Rouge de l'Est et 0,01 en Montbéliarde par exemple. L'allèle $S^{U U^{\prime \prime} H^{\prime}}$, en particulier, dont la fréquence est maximale en race Charolaise $(0,32)$, est rare en Montbéliarde $(0,04)$ et paraît absent en Pie-Rouge de l'Est. Par ailleurs, l'allèle $A^{A Z^{\prime}}$ couramment présent chez le zébu, mais absent dans toutes les races nordiques, atteint sa fréquence maximale en race Charolaise $(0,22)$, assez loin devant l'Aubrac $(0,13)$, la Ferrandaise $(0,08)$, la Blonde d'Aquitaine $(0,07)$ et contre moins de 0,01 en Montbéliarde et en Pie-Rouge de l'Est. On voit que pour des allèles qui contribuent à caractériser ce groupe, la Charolaise est même, de toutes les races du Centre et du Sud-Ouest, celle qui montre les différences les plus accusées avec les autres groupes. Bien entendu, cette conclusion ne peut exclure une certaine influence des races jurassiennes; de même, si la présence de l'allèle $B^{B O_{3} Y A^{\prime} E_{3}^{\prime} G^{\prime} P^{\prime} Q^{\prime} G^{\prime \prime} I^{\prime \prime}}$, accumulé en race Shorthorn $(0,34$ dans notre échantillon; 0,37 aux USA selon Rausch et al, 1967) par suite d'une forte sélection en consanguinité à ses «origines» (Robertson, 1956), est l'indice d'un apport Durham, la fréquence de cet allèle en race Charolaise $(0,14)$, identique à celle de la Maine-Anjou, atteste de la rémanence de gènes Durham. Toutefois, compte tenu des autres particularités alléliques de la race Charolaise, les influences jurassienne et britannique paraissent faibles. La seconde présence inattendue dans ce groupe est celle de la race Aubrac, que Sanson (1884) apparentait à la Parthenaise et Dechambre (1913) aux races «rectilignes» (Parthenaise, Bretonne, Brune, etc). Or, quelle que soit la méthode utilisée, l'Aubrac s'accole à la Salers, observation à rapprocher du fait que les aires d'extension de ces races sont contiguës et même partiellement imbriquées (Vissac, 1970). 
L'appartenance à un même groupe, dit de l'axe est-ouest, de races du Jura, des Alpes du Nord et de l'Ouest de la France peut également étonner. Ce résultat recoupe toutefois certaines des conclusions de Dechambre (1913) qui plaçait dans un même groupe (races «rectilignes») les races brunes, la Parthenaise et la Bretonne (fig 6). Plus récemment, Denis (1983) lançait l'idée que la Parthenaise pourrait «constituer la pointe occidentale de la progression du groupe brun des Alpes». Nos conclusions vont encore plus loin puisqu'elles font jouer ce rôle à la Bretonne Pie-Noire. Or ce résultat n'est pas nécessairement aberrant. En effet, le peuplement humain de l'Armorique s'est fait pour l'essentiel, du néolithique au dernier âge du fer (époque celtique), à partir du continent, le brassage des populations semblant d'ailleurs avoir été moins important que dans le Bassin parisien (Giot $e t$ $a l, 1979)$. Il ne saurait faire de doute que le peuplement bovin est également venu de l'Est, le trajet le plus court passant par la vallée de la Loire. Rien ne dit que les

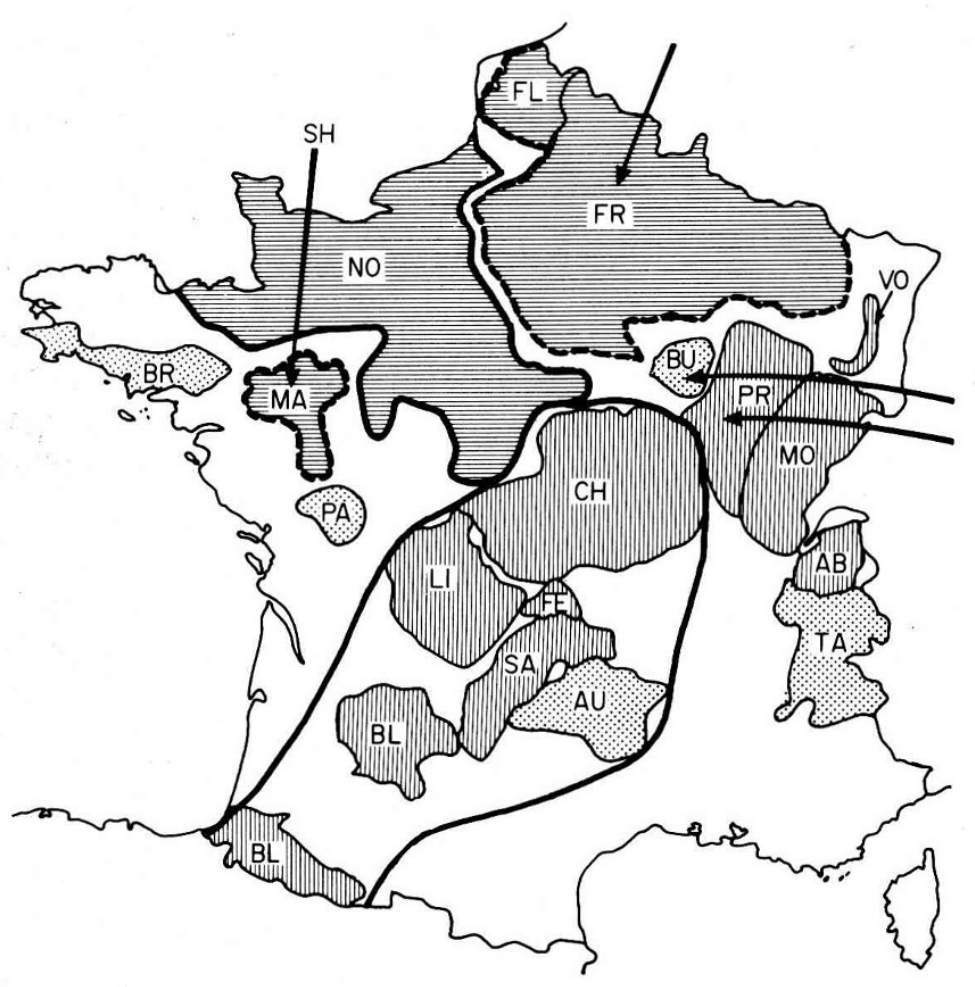

Fig 6. Zones d'implantation, vers 1960 (Quittet, 1963), des races considérées dans ce travail. Les contours épais délimitent 3 des 4 groupes raciaux mis en évidence: races du Nord (en tirets), races du Centre et du Sud-Ouest, race Normande. Les autres races constituent le groupe de l'axe est-ouest. Flèches = races d'origine étrangère. Hachures et grisé, groupes proposés par Dechambre (1913) sur la base de critères morphologiques: hachures horizontales : races «concavilignes»; hachures verticales: races «convexilignes »; grisé: races «rectilignes». 
infiltrations de Bretons venus d'Angleterre, du $\mathrm{V}^{\mathrm{e}}$ au VII $\mathrm{VI}^{\mathrm{e}}$ siècle de notre ère, se soient accompagnées d'un apport significatif de bovins (cf ci-dessus l'interrogation de même nature sur les origines de la Normande). D'ailleurs, selon Falc'hun (1970), il ne semble pas que ces infiltrations aient beaucoup affecté le Morbihan, considéré comme la zone d'origine de la race Bretonne Pie-Noire (Quittet, 1963) au point que le dialecte breton de cette région pourrait bien dériver du gaulois d'Armorique et non du britonnique insulaire. On notera toutefois, en comparant les dendrogrammes obtenus avec les différents indices, que les races périphériques du groupe de l'axe est-ouest, dont la Bretonne Pie-Noire, paraissent rattachées à ce groupe d'une manière plus lâche que d'autres; la Bretonne Pie-Noire (indice de Cavalli-Sforza) et la Vosgienne (Nei standard) montrent une certaine affinité avec le groupe nordique, la Parthenaise (Cavalli-Sforza) avec le groupe du Centre et du Sud-Ouest.

La race Normande occupe une place à part, due notamment à la forte fréquence de $\beta$ - $C n^{B}$, mais aussi de $F^{V}$, et à la présence de $M^{M_{1}}$. Ces caractéristiques tendent à la rapprocher de la race de Jersey (Larsen et al, 1974), non incluse dans le présent travail. L'allèle $\beta-C n^{B}$ parait également fréquent en race Hereford (Grosclaude et Mahé, résultats non publiés). L'extension de l'analyse phylogénétique aux races des îles anglo-normandes et britanniques permettrait de mieux préciser le statut de la race Normande.

Il est intéressant de rapprocher les conclusions de notre étude de celles du travail de Manwell et Baker (1980). Le groupe des races du Centre et du Sud-Ouest, qui compte d'ailleurs 2 races à longues cornes, l'Aubrac et la Salers (Epstein et Mason, 1984), correspond au groupe mixte primigenius-brachyceros de Manwell et Baker; de toutes les races françaises ce groupe paraît bien posséder le pool génique le plus proche de celui du foyer d'origine commun aux bovins et aux zébus (niveaux de fréquence relativement élevés de $A^{A Z^{\prime}}$, de $\beta-C n^{A^{2}}$, d'allèles du système $S$ déterminant le facteur $U$ ); le groupe de l'axe est-ouest correspond au groupe des races des hauteurs («Upland brachyceros»), désignation qui devient impropre si les races Parthenaise et Bretonne Pie-Noire sont bien des extensions de ce groupe; la race Normande serait la seule représentante du groupe des îles de la Manche et le groupe des races nordiques correspondrait, dans le classement de Manwell et Baker, aux rameaux Pie des Plaines et nordique. Il est remarquable que les conclusions de notre analyse phylogénétique s'articulent aussi étroitement avec celles de Manwell et Baker, alors que celles-ci découlent d'une approche indépendante, à partir de systèmes génétiques pour l'essentiel différents (2 locus communs seulement parmi les 13 de notre étude, et les 10 de celle de Baker et Manwell). Les conclusions des 2 études se renforcent donc mutuellement.

La fiabilité de nos résultats nous paraît également renforcée, dans les conclusions de l'analyge des corrélations entre allèles et composantes principales, par la similitude frappante entre les gradients de fréquence, de direction sensiblement nord-sud, observés pour 2 couples indépendants d'allèles, $C^{C_{1}}$ et $C^{C_{1}^{\prime \prime}}$ d'une part, $\beta-C n^{A^{2}}$ et $\beta-C n^{A^{1}}$ d'autre part. Il est aussi remarquable que, parmi les locus communs à notre travail et à celui d'Astolfi et al (1983) sur les races italiennes, les mêmes locus $(A, C$ et $S$ ) contribuent fortement, dans les 2 cas, à définir les composantes principales. Ceci incite à entreprendre une étude d'ensemble des populations bovines européennes en se basant sur une série commune d'allèles comprenant en priorité ceux qui se sont avérés les plus discriminants. 
Si l'on accepte les conclusions de Menozzi et al (1978) sur les populations humaines européennes, le principal gradient de fréquences observable actuellement est d'origine très ancienne puisqu'il remonte à l'expansion de l'agriculture au néolithique. Nos résultats sur les races bovines françaises donnent aussi un éclairage sur leur passé le plus ancien, en les rattachant au processus général d'extension et de diversification de la population bovine en Europe proposé par Manwell et Baker (1980). On aura aussi noté, au sein de chaque groupe, parfois même aux limites entre les groupes, le poids de la proximité géographique, attestant d'une continuité génétique qui n'est guère surprenante. Il est clair que cette continuité génétique reste décelable parce que les systèmes polymorphes utilisés ont sans doute été peu touchés par la sélection récente en faveur de standards ou d'aptitudes zootechniques. Ainsi le présent travail dresse-t-il une première esquisse d'une évolution plusieurs fois millénaire, dont l'étude mériterait d'être complétée par la prise en compte de toutes les races françaises et élargie à l'ensemble du domaine européen; elle pourrait aussi être approfondie par l'adjonction d'autres systèmes génétiques qui devrait permettre une analyse plus fine des situations particulières.

\section{REMERCIEMENTS}

Nous exprimons notre gratitude aux très nombreuses personnes qui ont contribué à la collecte et à l'analyse des échantillons, ou à leur interprétation : les éleveurs, les responsables des organismes d'élevage et leurs collaborateurs, les équipes des laboratoires de génétique biochimique et des groupes sanguins, en particulier MT Alaux, M Faugeras, G Houlier, MF Mahé et G Ruffet, sans oublier M Boitard, laboratoire de génétique factorielle et $B$ Bonaïti, station de génétique quantitative et appliquée, Jouy-en-Josas. Nous devons à CM Ann Baker, alors à l'université d'Adélaïde, Australie, GJ Nicholls, Milk Marketing Board, Thames Ditton, UK, RL Spooner et $\mathrm{D}$ Ross, Abro, Edimbourg, d'avoir eu accès à des échantillons de lait et de sang de la race Shorthorn. Nous remercions également $M$ Nei pour le don de programmes informatiques. F Grosclaude exprime sa reconnaissance à $\mathrm{A}$ Cauderon, de l'Académie des sciences, pour l'intérêt qu'il a porté à cette recherche. Ce travail a bénéficié d'une aide financière du ministère de l'Industrie et de la Recherche (Contrat DGRST n ${ }^{\circ} 81 \mathrm{G} 0934,1981-1983$ ).

\section{RÉFÉRENCES}

Ammerman A, Cavalli-Sforza LL (1971) A population model for the diffusion of early farming in Europe. In: The explanation of culture change (C Renfrew, ed) Duckworth, Londres, 1973, 343-357

Ashton GC (1965) Serum transferrin D alleles in Australian cattle. Austral J Biol Sci 18, 665-670

Astolfi P, Pagnacco G, Guglielmino-Matessi CR (1983) Phylogenetic analysis of native Italian cattle breeds. $Z$ Tierz ZüchtBiol 100, 87-100

Avon L (1989) Conservation et gestion des ressources génétiques bovines en France et en Europe occidentale. Institut Tech Elevage bovin, Paris, CR n ${ }^{\circ} 1652$ 
Baker CMA, Manwell C (1980) Chemical classification of cattle. 1. Breed groups. Anim Blood Groups Biochem Genet 11, 127-150

Bougler J, Le Liboux P (1973) Origine et historique de la race Charolaise. In: La race Charolaise, INRA, Département de Génétique animale, II, 1-22

Bouquet $Y$, Grosclaude $F$ (1968) Groupes sanguins et situation génétique de la race bovine flamande. Ann Biol Anim Biochim Biophys 8, 463-483

Cavalli-Sforza LL (1969) Human diversity. In: Proceedings of the XII International Congress of Genetics, Tokyo, 19-28 August 1968, Vol 3, 405-416

Ceppellini R, Siniscalco M, Smith CAB (1956) The estimation of gene frequencies in a random mating population. Ann Hum Genet 20, 97-115

Colleau JJ, Tanguy D, Boulanger P, Le Mezec P (1982) Prediction of diffusion of Holstein genes within the French Friesian population. In: Proceedings of the 2nd World Congress on Genetics applied to Livestock Production, Madrid, 4-8 October 1982, vol 8, Editorial Garsi, Madrid, 69-74

Dechambre P (1913) Traité de zootechnie. III. Les bovins. Ch Amat, Asselin et Houzeau, Paris

Denis B (1983) Parenté et filiation des races bovines françaises actuelles vues par les anciens auteurs. Ethnozootechnie 32, 141-158

Dolukhanov PM (1971) The neolithization of Europe: a chronological and ecological approach. In: The explanation of culture change (C Renfrew ed) Duckworth, Londres, 1973, 329-342

Epstein H (1971) The origin of the domestic animals of Africa, vol 1, Africana Publ Corp, New York

Epstein H, Mason IL (1984) Cattle. In: Evolution of domesticated animals. (IL Mason ed). Longman, Londres

Falc'hun F (1970) Les noms de lieux celtiques. Editions armoricaines, Rennes

Friend JB (1978) Cattle of the world. Blandford Press, Poole, Dorset

Giot PR, Briard J, Pape L (1979) Protohistoire de la Bretagne. Ouest-France, Rennes

González P, Tuñon MJ, Vallejo M (1987) Genetic relationships between seven Spanish native breeds of cattle. Anim Genet 18, 249-256

Gower JC (1966) Some distance properties of latent root and vector methods used in multivariate analysis. Biometrika $53,325-338$

Graml R, Schmid DO, Erhardt L, Buchberger J, Ohmayer G, Pirchner F (1986) Verwandtschaft des Murnau-Werdenfelser Rindes zu anderen Rassen. Bayer Landwirtsch Jahrb 63, 273-281

Gregorius HR (1984) A unique genetic distance. Biom J 26, 13-18

Grosclaude $F$ (1988) Le polymorphisme génétique des principales lactoprotéines bovines. INRA Prod Anim 1, 5-17

Grosclaude F, Millot P (1962) Contribution à l'étude des groupes sanguins de la race bovine Montbéliarde. Ann Biol Anim Biochim Biophys 2, 185-208

Grosclaude F, Mahé MF, Mercier JC (1974) Comparaison du polymorphisme génétique des lactoprotéines du Zébu et des bovins. Ann Génét Sél anim 6, 305-329

Grosclaude F, Guérin G, Houlier G (1979) The genetic map of the B system of cattle blood groups as observed in French breeds. Anim Blood Groups Biochem Genet 10, 199-212 
Grosclaude F, Lefebvre J, Noé G (1983) Nouvelles précisions sur la carte génétique du système de groupes sanguins B des bovins. Génét Sél Evol 15, 45-54

Guérin G, Grosclaude F, Houlier G (1981) The C system of cattle blood groups. 2. Partial genetic map of the system. Anim Blood Groups Biochem Genet 12, 15-21

Kidd KK (1969) Phylogenetic analysis of cattle breeds. $\mathrm{Ph} \mathrm{D}$ Thesis, Univ of Wisconsin

Kidd KK, Pirchner F (1971) Genetic relationships of Austrian cattle breeds. Anim Blood Groups Biochem Genet 2, 145-158

Kidd KK, Stone WH, Crimella C, Carenzi C, Casati M, Rognoni G (1982) Immunogenetic and population genetic analyses of Iberian cattle. Anim Blood Groups Biochem Genet 11, 21-38

De Lapparent C (1914) Etude sur les races, variétés et croisements de l'espèce bovine en France. Extrait, Bull Mens Office Rens Agric, Imprimerie nationale, Paris

Larsen B (1971) Blood groups and polymorphic proteins in cattle and swine. Ann Génét Sél Anim 3, 59-70

Larsen B, Gruchy CL, Moustgaard J (1974) Studies on blood groups and polymorphic protein systems in Jersey cattle on the isle of Jersey. Acta Agric Scand 24, 99-110

Lefebvre J (1983) Introduction aux analyses statistiques multidimensionnelles $\left(3^{\mathrm{e}}\right.$ ed) Masson, Paris

Lefebvre J, Boitard M, Rey JF (1981) Logiciels d'analyses statistiques multidimensionnelles sur micro-ordinateurs. Masson, Paris

Manwell C, Baker CMA (1980) Chemical classification of cattle. 2. Phylogenetic tree and specific status of the Zebu. Anim Blood Groups Biochem Genet 11, 151-162

Menozzi P, Piazza A, Cavalli-Sforza L (1978) Synthetic maps of human gene frequencies in Europeans. Science 201, 786-792

Nei M (1972) Genetic distance between populations. Am Nat 106, 283-292

Nei M (1973) The theory and estimation of genetic distance. In: Genetic structure of populations. (NE Morton, ed) Univ Hawaï Press, Honolulu

Nei M, Tajima F, Tateno Y (1983) Accuracy of estimated phylogenetic trees from molecular data. II. Gene frequency data. J Mol Evol 19, 153-170

Quittet E (1963) Races bovines françaises. La Maison rustique, Paris ( $\left.2^{\mathrm{e}} \mathrm{ed}\right)$

Quittet E, Blanc H (1974) Races chevalines en France. La Maison rustique, Paris

Rausch WH, Hines HC, Brum EW (1967) A blood type comparison of three beef breeds in America. Immunogen Lett 5, 43-49

Robertson A (1956) Blood grouping in dairy cattle improvement. In: Proc of the VII Int Cong Anim Husbandry, Madrid, May 1956, II, 79-86

Sanson A (1884) Traité de Zootechnie. IV. Zoologie et zootechnie spéciales; Bovidés taurins et Bubalins. Libr agric Maison rustique, Paris $\left(3^{\mathrm{e}} \mathrm{ed}\right)$

Sarrazin M (1962) La race bovine normande. Desseaux et Fils, Colombes

Sneath PHA, Sokal RR (1973) Numerical taxonomy. WH Freeman, San Francisco

Vernier M (1953) Historique de la race Montbéliarde. Camponovo, Besançon

Vissac B (1970) Etude génétique de la race d'Aubrac. In: L'Aubrac, CNRS, Paris, I, 29-102 